\title{
Occurrence of Human Cytomegalovirus Glycoprotein B Genotypes in Immunocompetent and Immunosuppressed Taiwanese Patients
}

\author{
Keh-Gong Wu $\mathrm{u}^{\mathrm{a}, \mathrm{b}}$ Miao-Chiu Hung ${ }^{\mathrm{a}, \mathrm{b}}$ Ya-Ting Chang ${ }^{c} \quad$ Chun-Jen Chen ${ }^{\mathrm{a}, \mathrm{b}}$ \\ Su-Pen Yang b, d Cheng-Yi Liu ${ }^{\text {b, d }}$ Donald Ming-Tak Hob, e Yu-Jiun Chan b, c, f \\ ${ }^{a}$ Department of Pediatrics, Taipei Veterans General Hospital, b School of Medicine, National Yang-Ming University, \\ ' Institute of Public Health, National Yang-Ming University, ${ }^{d}$ Division of Infectious Diseases, Department of Medicine, \\ eDepartment of Pathology and Laboratory Medicine, and ${ }^{\mathrm{f}}$ Division of Virology, Department of Pathology and \\ Laboratory Medicine, Taipei Veterans General Hospital, Taipei, Taiwan, ROC
}

\section{Key Words}

Human cytomegalovirus • Glycoprotein B · Genotypes • Immunocompetence $\cdot$ Immunosuppression - Taiwanese

\begin{abstract}
Objectives: To investigate the differences of genotypic distributions among isolates between immunosuppressed and immunocompetent patients in a Taiwanese population. Methods: Human cytomegalovirus (HCMV) isolates from 76 patients with adequate chart data were analyzed. Polymerase chain reaction-restriction fragment length polymorphism (PCR-RFLP) was used to define gB genotypes which include gB1 to gB4. The clinical data of the 76 patients were retrospectively collected by chart review and classified into an immunosuppressed $(n=32)$ or immunocompetent $(n=$ 44) group. Results: Among the 32 immunosuppressed patients, the most commonly identified HCMV genotypes were gB1 (27/32, 84.3\%) and gB3 (4/32, 12.5\%). 59.1\% (26/44) of the immunocompetent patients were infected by $\mathrm{gB} 1$ while $38.6 \%(17 / 44)$ of them were infected by gB3. The frequency of $\mathrm{gB} 1$ infection in the immunosuppressed group was significantly higher than that in the immunocompetent group $(p=0.025)$. However, there was no statistically significant dif-
\end{abstract}

ference between gB1 and gB3 distributions by clinical diagnosis within each group. Conclusions: Only gB1 and gB3 genotypes were identified in this Taiwanese population. Although there is no significant difference between clinical diagnosis and gB genotyping, gB1 infection is significantly more predominant in immunosuppressed patients.

Copyright $\odot 2011$ S. Karger AG, Basel

\section{Introduction}

Human cytomegaloviruses (HCMV), members of the Herpesviridae family, have large (220-240 kbps) and complex viral genomes composed of double-stranded DNA $[1,2]$. This important pathogen is capable of establishing HCMV diseases, ranging from asymptomatic to life-threatening.

K.-G.W. and M.-C.H. contributed equally to this article. This research was presented in part at the 11th Western Pacific Congress of Chemotherapy and Infectious Diseases (WPCCID), Taipei, Taiwan, ROC, December 2, 2008, PS3-012.

\section{KARGER}

Fax +4161306 1234

E-Mail karger@karger.ch

www.karger.com
(C) 2011 S. Karger AG, Basel

0300-5526/11/0544-0196\$38.00/0

Accessible online at:

www.karger.com/int
Yu-Jiun Chan, MD, PhD

Division of Virology, Department of Pathology and Laboratory Medicine Taipei Veterans General Hospital, No. 201, Section 2

Shih-Pai Road, Taipei 11247, Taiwan (ROC)

Tel. +88622875 7297, Fax +88622831 1311, E-Mail yjchan@vghtpe.gov.tw 
HCMV diseases are related to host immune status. Congenitally infected babies can be born with 'cytomegalic inclusion disease' or have asymptomatic infections, while some sequelae were noted during follow-up. Among immunocompetent hosts, HCMV infection causes asymptomatic infection, fever, infectious mononucleosis, or acute viral hepatitis. For immunosuppressed hosts, acute HCMV infection also causes a wide spectrum of disease involving specific organs or causing constitutional disturbances. In recipients of bone marrow grafts, pneumonitis is the major fatal disease of HCMV infections. HCMV retinitis, esophagitis, colitis, encephalitis, and polyradiculopathy are diseases that commonly occur among patients with AIDS [3].

The HCMV genome encodes numerous glycoproteins, of which $\mathrm{gB}, \mathrm{gH}$ and $\mathrm{gN}$ are the most abundant. The $\mathrm{gB}$, $\mathrm{gH}$, and gN genes are all utilized for genotyping [1]. Many genetically different strains of HCMV exist within the human population.

Glycoprotein B is essential for both in vivo and in vitro viral replication and it also plays a role in viral-host interaction. The polymorphisms of $\mathrm{gB}$ interfere with HCMV pathogenesis. Experimental evidence showed that different viral strains are associated with differing abilities to replicate in endothelial and smooth muscle cells, and have differing abilities to cause immunosuppression. Nevertheless, the exact mechanisms by which these events occur remain unknown [4].

Glycoprotein B is encoded by the ORF UL55 gene, with several genetic polymorphisms. There are four major gB genotypes ( $\mathrm{gB1}$ to gB4) and additional non-prototypic genotypes are also described [5].

Many studies widely investigated the association between different $\mathrm{gB}$ genotypes and their virulence, viral cell tropism, clinical manifestations, and outcomes [620]. Nevertheless, previous studies pointed out inconsistent results regarding different $\mathrm{gB}$ subtypes and their distributions. For example, gB1 is related to acute graft rejection in transplant recipients [2]. Woo et al. [6] and Lukacsi et al. [21] found a high prevalence of gB1 in congenital infections. In contrast, Yan et al. [12] found that gB3 genotype was more prevalent in congenital infections than in postnatally infected children. Additionally, mixed infections with multiple gB genotypes are associated with severe clinical manifestations $[22,23]$.

Although a previous study [24] indicated that only gB1 and $\mathrm{gB} 3$ were detected among Taiwanese patients, there are no data regarding $\mathrm{gB}$ genotypes and their clinical manifestations. Therefore, this study was performed to correlate $\mathrm{gB}$ genotypes with clinical disease presentations.

\section{Materials and Methods}

\section{Samples}

A total of 125 clinical samples from 113 patients were identified as HCMV-positive by viral culture in Taipei Veterans General Hospital from 2005 to 2006. All the isolates were stored at $-70^{\circ}$. Among the 12 extra samples, there were 4 repeated samples from a serial follow-up of a patient, 2 from another 3 patients, and 1 from the other 2 patients.

Clinical specimens included throat swabs $(n=48)$, urine $(n=$ $18)$, bronchoalveolar lavage $(n=7)$, and others $(n=3)$ including peritoneal dialysate, blood and vitreous fluid, respectively.

Because not all cultivatable viruses replicated in a single cell line, all clinical specimens sending to virology laboratory were immediately inoculated into four different cell culture media simultaneously including MRC-5 (human lung embryonal fibroblast cells), MDCK (Madin-Darby canine kidney cells), HEp-2 (continuous human epidermoid cancer cells) and RD (human rhabdomyosarcoma cells).

\section{Review of Charts}

Of the 113 patients, 85 had sufficiently complete chart data to be included in the analysis. Patient age, sex, underlying diseases, and clinical diagnosis were recorded. For further data analysis, patients were classified into two groups: the immunosuppressed group included those with hematological malignancies with or without stem cell transplantation, post-solid organ transplantation, autoimmune diseases treated with immunosuppressant agents. Immunocompetent hosts were defined as patients without underlying documented immunodeficiency or immunosuppression. Since congenital HCMV infection was considered as a different disease entity, patients $<3$ months of age $(n=9)$ were excluded. Thus, there were 76 patients analyzed in this study.

Ethical approval for this study was granted by the Human Investigation Review Board of the Taipei Veteran General Hospital.

\section{Polymerase Chain Reaction-Restriction Fragment Length Polymorphism}

HCMV-DNA was extracted from frozen clinical isolates using the Qiamp Blood Mini Kit (Qiagen Inc., Chatsworth, Calif., USA) according to the manufacturer's recommendations. DNA samples were resuspended in $200 \mu$ l of diethylpyrocarbonate-treated water. The amplification reaction was performed according to the following protocol. Oligonucleotide primers, each with a concentration of $1.5 \mu \mathrm{M} / \mu \mathrm{l}$, were added to the polymerase chain reaction (PCR) mixture. The latter contained $27.75 \mu \mathrm{l}$ of triple distilled $\mathrm{H}_{2} \mathrm{O}, 10 \mu \mathrm{l}$ of $5 \times$ Go Taq buffer, $3 \mu \mathrm{l}$ of $\mathrm{MgCl}_{2}(25 \mathrm{~mm}), 1 \mu \mathrm{l}$ of $10 \mathrm{mM}$ each dNTP, and $1.25 \mathrm{U}$ Taq DNA polymerase per $50 \mu \mathrm{l}$ of reaction mixture. The primers that we used were gB1319 (TGGAACTGGAACGTTTGGC) and gB1604 (GAAACGCGCGGCAATCGG). The amplified PCR product was about $300 \mathrm{bp}$ [25]. The reaction mixture consisted of $45-\mu l$ aliquots and $5-\mu l$ DNA samples. PCR was as the following: initial denaturation at $95^{\circ}$ for $6 \mathrm{~min}$, denaturation at $95^{\circ}$ for $5 \mathrm{~min}$, annealing at $55^{\circ}$ for $2 \mathrm{~min}$ and extension at $72^{\circ}$ for $1 \mathrm{~min}$. A final extension was performed at $72^{\circ}$ for 7 min. DNA from the HCMV AD169 strain was used as the positive control and normal human plasma was used as a negative control.

For the identification of $g B$ genotypes, an aliquot of the amplified product was digested with the restriction enzymes HinfI and 
RsaI. The respective genotypes were determined by restriction fragment length polymorphism (RFLP) analysis. Restriction fragments were detected by electrophoresis on $2 \%$ agarose gels. The gels were stained with ethidium bromide and viewed on an ultraviolet transilluminator. The HCMV clinical isolates were then classified as gB1 to gB4 [25].

\section{Sequencing}

If RFLP failed to differentiate the genotype, the PCR products were subjected to sequencing analysis (7 isolates).

\section{Statistical Analysis}

Correlation analyses to correlate HCMV gB genotypes and patient age, sex, clinical diagnosis, and host immune status were conducted. The $\chi^{2}$ method was applied, and $\mathrm{p}$ values $<0.05$ were considered statistically significant.

\section{Results}

\section{Demographic Data}

Among the 76 patients with sufficient clinical information, 32 patients were grouped as immunosuppressed while the other 44 were in immunocompetent group. There were 27 female and 49 male patients. As to age distribution, $97.7 \%$ (43/44) of the immunocompetent patients were $<16$ years. In contrast, only $18.8 \%(6 / 32)$ of immunosuppressed patients were $<16$ years. A statistically significant difference $(\mathrm{p}<0.01)$ was noted.

\section{Sequencing and Genotyping Results}

The sequencing results showed that all the 7 isolates that could not be digested by RsaI belonged to gB1 genotype. Serial samples from the same patient all had the same genotyping results. Therefore, only one genotyping result for each patient was counted.

Among the 76 patients, 53 were infected by gB1 (69.7\%), 21 by gB3 (27.6\%), and 2 mixed gB1 and gB3 (2.6\%). The most common $\mathrm{gB}$ genotype in the immunosuppressed group was gB1 $(27 / 32,84.3 \%)$, followed by gB3 (4/32, $12.5 \%)$. For the immunocompetent patients, the most common genotype was gB1 $(26 / 44,59.1 \%)$, followed by gB3 (17/44, 38.6\%). The frequency of HCMV gB1 was significantly higher in the immunosuppressed group than in the immunocompetent group ( $\mathrm{p}=0.025)$.

The distributions of $\mathrm{gB}$ genotypes in different types of specimens are shown in table 1 . Most specimens were from throat swab or sputum $(48 / 76,63.2 \%)$. There were no statistically significant differences between the distributions of gB genotypes from different specimens.

The distribution of $\mathrm{gB}$ genotypes among different underlying diseases in immunosuppressed patients is shown
Table 1. Glycoprotein B1 (gB1) and B3 (gB3) distributions by specimen type

\begin{tabular}{lccl}
\hline & gB1 & gB3 & $\begin{array}{l}\text { Mixed gB1 } \\
\text { and gB3 }\end{array}$ \\
\hline Urine $(\mathrm{n}=18)$ & $14 / 18$ & $3 / 18$ & $1 / 18$ \\
Throat swab/sputum $(\mathrm{n}=48)$ & $30 / 48$ & $17 / 48$ & $1 / 48$ \\
Bronchoalveolar lavage $(\mathrm{n}=7)$ & $6 / 7$ & $1 / 7$ & - \\
Others $^{1}(\mathrm{n}=3)$ & $3 / 3$ & - & - \\
\hline
\end{tabular}
(1)

${ }^{1}$ Others: peritoneal dialysate (1), blood (1), and vitreous fluid

Table 2. Glycoprotein B1 (gB1) and B3 (gB3) genotype frequencies by underlying immunosuppressive disease group $(\mathrm{n}=32)$

\begin{tabular}{|c|c|c|c|}
\hline & $\mathrm{gB} 1$ & gB3 & $\mathrm{gB} 1+\mathrm{gB} 3$ \\
\hline Post-autologous SCT $(\mathrm{n}=5)$ & $5 / 5$ & - & - \\
\hline Post-allogeneic SCT $(\mathrm{n}=15)$ & $12 / 15$ & $2 / 15$ & $1 / 15$ \\
\hline Malignancy without SCT $(\mathrm{n}=5)$ & $5 / 5$ & - & - \\
\hline Post-solid organ transplantation $(\mathrm{n}=1)$ & - & $1 / 1$ & - \\
\hline $\begin{array}{l}\text { Acquired immunodeficiency syndrome } \\
\qquad(\mathrm{n}=1)\end{array}$ & $1 / 1$ & - & - \\
\hline Other immunosuppressive status ${ }^{1}(n=5)$ & $4 / 5$ & $1 / 5$ & - \\
\hline Total $(\mathrm{n}=32)$ & $27 / 32$ & $4 / 32$ & $1 / 32$ \\
\hline \multicolumn{4}{|c|}{$\begin{array}{l}\text { SCT }=\text { Stem cell transplantation. } \\
1 \text { Other immunosuppressive status included systemic lupus } \\
\text { ythematosus, chronic obstructive pulmonary disease, idio- } \\
\text { thic thrombocytopenic purpura status post-splenectomy and } \\
\text { ult-onset Still's disease. }\end{array}$} \\
\hline
\end{tabular}

in table 2. The most common underlying immunosuppressive disease in this study is post-stem cell transplantation $(\mathrm{n}=20)$ among which $85 \%(17 / 20)$ were infected by gB1.

Clinical diagnoses of both the immunosuppressed and immunocompetent groups and their gB distributions are shown in table 3 . There were 2 patients who were diagnosed as CMV hepatitis, 1 in each group. Both patients were admitted due to fever, poor appetite and malaise. Mild hepatomegaly on physical examination in association with elevated hepatic enzyme levels was found, viral hepatitis was initially impressed. A presumptive diagnosis of CMV hepatitis was made later on the basis of positive CMV culture in urine specimens and a fourfold increase of $\mathrm{CMV}$-specific IgG antibody titer in paired sera. The only 1 patient with CMV retinitis in our case 
Table 3. Glycoprotein B1 (gB1) and B3 (gB3) distributions by clinical diagnosis within the immunosuppressed and immunocompetent groups

\begin{tabular}{lccl}
\hline & gB1 & gB3 & $\begin{array}{l}\text { Mixed gB1 } \\
\text { and gB3 }\end{array}$ \\
\hline Immunosuppressed group $(\mathrm{n}=32)$ & $27 / 32$ & $4 / 32$ & - \\
CMV pneumonitis $(\mathrm{n}=10)$ & $8 / 10$ & $2 / 10$ & - \\
CMV retinitis $(\mathrm{n}=1)$ & $1 / 1$ & - & - \\
CMV hepatitis $(\mathrm{n}=1)$ & $1 / 1$ & - & - \\
Hemorrhagic cystitis $(\mathrm{n}=1)$ & - & - & $1 / 1$ \\
Other unspecified infection $(\mathrm{n}=19)$ & $17 / 19$ & $2 / 19$ & - \\
\hline Immunocompetent group $(\mathrm{n}=44)$ & $26 / 44$ & $17 / 44$ & $1 / 44$ \\
Lower respiratory tract infection & $11 / 17$ & $6 / 17$ & - \\
Upper respiratory tract infection ${ }^{1}$ & $13 / 18$ & $5 / 18$ & - \\
CMV hepatitis & - & $1 / 1$ & - \\
Other unspecified infection & $2 / 7$ & $5 / 7$ & - \\
\hline
\end{tabular}

${ }^{1}$ Among the 18 patients diagnosed with upper respiratory tract infections, 3 had hand, foot and mouth disease, and 7 had herpangina.

series was a 33-year-old female suffering from systemic lupus erythematosus for many years. She was once admitted to the ophthalmic ward due to rapidly progressive decrease of visual acuity in her right eye for 3 weeks. CMV retinitis was diagnosed on the basis of ophthalmoscopic examination showing a macular hole with retinal necrosis, and positive CMV culture from a vitreous fluid specimen. Intravenous ganciclovir was given for 2 weeks followed by one dose of intravitreal injection of ganciclovir with uneventful outcome. Among the 18 patients diagnosed as the upper respiratory tract infection, 3 had clinical diagnosis of hand, foot and mouth disease and another 7 were diagnosed as herpangina. Nevertheless, there was no statistically significant difference between $\mathrm{gB} 1$ and $\mathrm{gB} 3$ distributions by clinical diagnosis within the immunosuppressed $(\mathrm{p}=0.84)$ and immunocompetent $(\mathrm{p}=0.128)$ groups.

\section{Discussion}

This study showed that only the gB1 and gB3 genotypes were detected in this Taiwanese subpopulation. The geographic distribution of $\mathrm{gB}$ genotypes that we report is similar to that previously reported in Japan [9]. Nonetheless, all four major gB genotypes were detected among infants with congenital infections in mainland China [10]. In comparison to studies in Italy, the predominant HCMV strains encountered were gB2 and gB3 [13]. In Thailand, Bhattarakosol and Chantaraarphonkun [20] found that mixed infections (35\%) were high in HIV-infected patients. Glycoprotein B1 (33\%) predominates in Thai patients and no gB4 was detected. In different patient populations in Costa Rica, gB2 was the most frequent gB genotype encountered (73\%) [15]. In a Cuban cohort of patients with different HCMV diseases, gB2 was the most prevalent (38\%), followed by gB1 (30\%), and mixed HCMV infections (16\%) [8]. These differences reveal variations in the geographical distributions of global HCMV strains.

Among the patients we studied, mixed infections with $\mathrm{gB} 1$ and $\mathrm{gB} 3$ only occurred in 2 patients. One of them was immunosuppressed, while the other was immunocompetent. Previous reports indicated that mixed infections were more prevalent among immunosuppressed hosts $[8$, $22]$. However, our findings did not concur previous reports by Yan et al. [12] and Coaquette et al. [22].

This study showed no statistical difference of gB distribution within each group by clinical diagnoses. In contrast, a previous study in bone marrow transplant recipient patients showed that shedding of gB1 seemed to have a better outcome. Our patient population was more heterogeneous and the patient number in each diagnosis group was also relatively small. Nevertheless, the previous study indicated no significant predominance of a specific gB genotype even within a homogeneous population with congenital CMV disease [26].

Because more pediatric patients were included in this study (49/76, 64\%), age as a confounding factor should be examined carefully. Firstly, the reason why most samples were obtained from pediatric patients is associated with clinical practice. In this hospital, only immunosuppressed adults, especially those who had stem cell or solid organ transplantations, would have been sampled (either from urine or bronchoalveolar lavage) for virus culture regularly. In contrast, virus cultures were commonly used for investigation of fevers in pediatric patients, who constituted most of the immunocompetent group. Secondly, there were no previous studies addressing the genotypic distribution among different age groups. Since a great proportion of the HCMV diseases in adults were due to reactivation, genotypic transition in adulthood was less likely. Finally, though there was no statistically significance of gB1 distribution between pediatric immunosuppressed patients $(6 / 6,100 \%)$ and immunocompetent children $(25 / 43,58.1 \%)$, the trend of greater proportion of $\mathrm{gB} 1$ distribution among immunosuppressed children is a consistent finding. 
Most specimens among the immunocompetent group in this study were isolated from throat swabs (63.2\%). There were no statistically significant differences between the distributions of gB genotypes from different specimens. Almost all of these patients had respiratory tract infections, including acute pharyngotonsillitis, acute bronchiolitis, and bronchopneumonia.

Virus isolation from throat swabs of a few patients with diagnoses of herpangina yielded HCMV. Although HCMV seldom causes acute pharnygitis, it is still a mong the list of the etiologic agents of pharyngitis. In immunocomptent patients, HCMV can cause a mononucleosis-like syndrome, the most common manifestations are fever, pharyngitis, lymphadenopathy, fatigue and hepatosplenomegaly, but early in its course, this disorder can be difficult to distinguish from other causes of pharyngitis [3]. Hence it was very likely that a pediatrician might occasionally mistake a HCMV-induced pharyngitis for a herpangina-like illness due to enterovirus. Mixed infection of enterovirus and HCMV is also possible [27].

Intra- and intergenotypic variations among HCMV gB genotypes were previously studied [28-30]. Although PCR-RFLP is not sufficiently sensitive to differentiate all the variants of HCMV genotypes, its combination with subsequent sequencing is still feasible due to cost-effectiveness.
We recognize several limitations to our study, including its retrospective nature and the small number of patients studied. Combining PCR for HCMV in patient blood samples, serum anti-HCMV IgM, or a fourfold elevation of anti-HCMV IgG titer supports the diagnosis of HCMV disease better than virus culture alone. Viral culture takes several days to 1 month to yield a final report of HCMV. During acute illnesses, routinely taking blood samples from every febrile patient is not feasible. A prospective, multicenter study using PCR to detect HCMV DNA in blood samples is needed to clarify the population-wide distribution of $\mathrm{gB}$ genotypes in the Taiwanese population.

In summary, this study showed that only gB1 and gB3 were identified in a Taiwanese population. Despite no statistical difference of $g B$ distribution within each group by clinical diagnoses, the frequency of gB1 was significantly higher in immunosuppressed than immunocompetent patients. Since age may be a confounding factor, further prospective study regarding the implications for outcome among the different HCMV gB genotypes is needed.

\section{Acknowledgement}

Part of this work was supported by VGH94-361, V96S22-008, V96C1-172 and V98C1-110 projects.

\section{References}

1 Dar L: Identifying human cytomegalovirus genotypes and defining their clinical significance. Indian J Med Res 2007;126:99-100.

-2 Pignatelli S, Dal MP, Rossini G, Landini MP: Genetic polymorphisms among human cytomegalovirus wild-type strains. Rev Med Virol 2004;14:383-410.

3 Crumpacker CS, Wadhwa S: Cytomegalovirus; in Mandell GL, Bennett JE, Dolin R (eds): Mandell, Douglas, and Bennett's Principles and Practice of Infectious Diseases, ed 6. Philadelphia, Churchill Livingstone, 2005, pp 1786-1798.

4 Rasmussen L: Molecular pathogenesis of human cytomegalovirus infection. Transpl Infect Dis 1999;1:127-134.

-5 Shepp DH, Match ME, Lipson SM, Pergolizzi RG: A fifth human cytomegalovirus glycoprotein B genotype. Res Virol 1998;149:109114.
6 Woo PC, Lo CY, Lo SK, Siau H, Peiris JS, Wong SS, Luk WK, Chan TM, Lim WW, Yuen KY: Distinct genotypic distributions of cytomegalovirus envelope glycoprotein in bone marrow and renal transplant recipients with cytomegalovirus disease. Clin Diagn Lab Immunol 1997;4:515-518.

-7 Steininger C, Schmied B, Sarcletti M, Geit M, Puchhammer-Stockl E: Cytomegalovirus genotypes present in cerebrospinal fluid of HIV-infected patients. AIDS 2005;19:273278.

-8 Kouri V, Gonzalez EE, Martinez PA, Capo V, Gonzalez R, Perez L, Viera J, Cardella L, Hengge UR: Distinct genotypic distribution of cytomegalovirus envelope glycoprotein B in a Cuban cohort of patients with different cytomegalovirus diseases. Scand J Infect Dis 2007;39:1038-1044.

-9 Kashiwagi Y, Kawashima H, Matsuura K, Sasamoto M, Takekuma K, Hoshika A, Nozaki-Renard J: Clinical characteristics and $\mathrm{gB}$ genotype of cytomegalovirus infection in Japan. In Vivo 2002;16:447-450.
10 Yu ZS, Zou CC, Zheng JY, Zhao ZY: Cytomegalovirus gB genotype and clinical features in Chinese infants with congenital infections. Intervirology 2006;49:281-285.

- 11 Yamamoto AY, Mussi-Pinhata MM, de Deus Wagatsuma VM, Marin LJ, Duarte G, Figueiredo LT: Human cytomegalovirus glycoprotein B genotypes in Brazilian mothers and their congenitally infected infants. J Med Virol 2007;79:1164-1168.

12 Yan H, Koyano S, Inami Y, Yamamoto Y, Suzutani T, Mizuguchi M, Ushijima H, Kurane $\mathrm{I}$, Inoue $\mathrm{N}$ : Genetic variations in the $\mathrm{gB}$, UL144 and UL149 genes of human cytomegalovirus strains collected from congenitally and postnatally infected Japanese children. Arch Virol 2008;153:667-674.

-13 Arista S, De GS, Giammanco GM, Di CP, Iannitto E: Human cytomegalovirus glycoprotein $\mathrm{B}$ genotypes in immunocompetent, immunocompromised, and congenitally infected Italian populations. Arch Virol 2003; 148:547-554 
14 Al-Ahdal MN, Frayha HH, Al-Thawadi SI, Kessie G: Typing of human cytomegalovirus clinical isolates from Saudi patients by PCRRFLP. Infection 2005;33:73-76.

15 Humada-Ruiz S, Taylor-Castillo L, Visona K, Luftig RB, Herrero-Uribe L: Determination of human cytomegalovirus genetic diversity in different patient populations in Costa Rica. Rev Inst Med Trop Sãa Paulo 2004;46:87-92.

16 Xanthakos SA, Schleiss MR: Glycoprotein B genotyping of cytomegalovirus strains isolated in a pediatric population. Pediatr Infect Dis J 2003;22:462-463.

17 Tanaka K, Numazaki K, Tsutsumi H: Human cytomegalovirus genetic variability in strains isolated from Japanese children during 1983-2003. J Med Virol 2005;76:356360 .

$\rightarrow 18$ Rasmussen L, Hong C, Zipeto D, Morris S, Sherman D, Chou S, Miner R, Drew WL, Wolitz R, Dowling A, Warford A, Merigan TC: Cytomegalovirus gB genotype distribution differs in human immunodeficiency virus-infected patients and immunocompromised allograft recipients. J Infect Dis 1997; 175:179-184.
19 Bale JF Jr, Murph JR, Demmler GJ, Dawson J, Miller JE, Petheram SJ: Intrauterine cytomegalovirus infection and glycoprotein B genotypes. J Infect Dis 2000;182:933-936.

20 Bhattarakosol P, Chantaraarphonkun S: Prevalence of human cytomegalovirus $\mathrm{gB}$ genotypes in Thai patients. Southeast Asian J Trop Med Public Health 2007;38:835-840.

21 Lukacsi A, Tarodi B, Endreffy E, Babinszki A, Pal A, Pusztai R: Human cytomegalovirus $\mathrm{gB}$ genotype 1 is dominant in congenital infections in South Hungary. J Med Virol 2001; 65:537-542.

22 Coaquette A, Bourgeois A, Dirand C, Varin A, Chen W, Herbein G: Mixed cytomegalovirus glycoprotein $B$ genotypes in immunocompromised patients. Clin Infect Dis 2004; 39:155-161.

23 Aquino VH, Figueiredo LT: High prevalence of renal transplant recipients infected with more than one cytomegalovirus glycoprotein B genotype. J Med Virol 2000;61:138142.

24 Chan YJ, Wu YW, Liu CY, Liu YC: Identification of two major human cytomegalovirus gB genotypes in Taiwan (ECCMID ). 15th European Congress of Clinical Microbiology and Infectious Diseases, Copenhagen 2005.
25 Chou SW, Dennison KM: Analysis of interstrain variation in cytomegalovirus glycoprotein $B$ sequences encoding neutralization-related epitopes. J Infect Dis 1991;163: 1229-1234.

26 Barbi M, Binda S, Caroppo S, Primache V, Dido P, Guidotti P, Corbetta C, Melotti D: CMV gB genotypes and outcome of vertical transmission: study on dried blood spots of congenitally infected babies. J Clin Virol 2001;21:75-79.

27 Shah VA, Chong CY, Chan KP, Ng W, Ling AE: Clinical characteristics of an outbreak of hand, foot and mouth disease in Singapore. Ann Acad Med Singapore 2003;32:381-387.

-28 Chantaraarphonkun S, Bhattarakosol P: Intra- and intergenotypic variations among human cytomegalovirus gB genotypes. Intervirology 2007;50:78-84.

29 Rasmussen L, Geissler A, Winters M: Interand intragenic variations complicate the molecular epidemiology of human cytomegalovirus. J Infect Dis 2003;187:809-819.

30 Novak Z, Ross SA, Patro RK, Pati SK, Kumbla RA, Brice S, Boppana SB: Cytomegalovirus strain diversity in seropositive women. J Clin Microbiol 2008;46:882-886. 